\title{
The Psychoimmunology of Lyme/Tick-Borne Diseases and its Association with Neuropsychiatric Symptoms
}

\author{
Robert C. Bransfield*
}

225 Highway \#35, Red Bank, NJ 07701, USA

\begin{abstract}
Disease progression of neuropsychiatric symptoms in Lyme/tick-borne diseases can be better understood by greater attention to psychoimmunology. Although there are multiple contributors that provoke and weaken the immune system, infections and persistent infections are significant causes of pathological immune reactions. Immune mediated effects are a significant contributor to the pathophysiological processes and disease progression. These immune effects include persistent inflammation with cytokine effects and molecular mimicry and both of these mechanisms may be present at the same time in persistent infections. Sickness syndrome associated with interferon treatment and autoimmune limbic encephalopathies are models to understand inflammatory and molecular mimicry effects upon neuropsychiatric symptoms. Progressive inflammatory reactions have been proposed as a model to explain disease progression in depression, psychosis, dementia, epilepsy, autism and other mental illnesses and pathophysiological changes have been associated with oxidative stress, excitotoxicity, changes in homocysteine metabolism and altered tryptophan catabolism. Lyme disease has been associated with the proinflammatory cytokines IL-6, IL-8, IL-12, IL-18 and interferon-gamma, the chemokines CXCL12 and CXCL13 and increased levels proinflammatory lipoproteins. Borrelia burgdorferi surface glycolipids and flagella antibodies appear to elicit anti-neuronal antibodies and anti-neuronal antibodies and Borrelia burgdorferi lipoproteins can disseminate from the periphery to inflame the brain. Autism spectrum disorders associated with Lyme/tick-borne diseases may be mediated by a combination of inflammatory and molecular mimicry mechanisms. Greater interaction is needed between infectious disease specialists, immunologists and psychiatrists to benefit from this awareness and to further understand these mechanisms.
\end{abstract}

Keywords: Immune, inflammation, Lyme, mental, psychiatric, tick-borne.

\section{INTRODUCTION}

There are different degrees of evidence that infections and the immune reactions to them can cause degenerative neurological disease, mental illness, cognitive decline, developmental disabilities, personality changes and violence and the pathophysiology needs better clarification [1-7]. Disease can result from an interaction of predisposing and precipitating factors [5]. When diseases are associated with infection, the infection is followed by immune and other reactions that can lead to a pathophysiological process resulting in dysfunction leading to symptoms and syndromes of dysfunction [5]. Ineffective treatment then can result in further disease progression [8].

Understanding the interaction between the immune system and nervous systems is critical however psychiatrists have little training and experience in immunology and immunologists have little training and experience dealing with psychiatry. However, the brain and immune systems have many similarities - both defend against threats by shifting allocation of resources as environments change; both have intracellular transmitters, receptors and feedback capability;

*Address correspondence to this author at the 225 Highway \#35, Red Bank, NJ 07701, USA; Tel: 732-741-3263; Fax: 732-741-5308;

E-mails: brancfield@comcast.net; rbrancfield@gmail.com there are similarities between the gut and immune barrier and the bold brain barrier (BBB); both have innate and learned capabilities and in both cases failures to shift from innate to learned responses result in pathology. [9] The brain and immune systems both switches back and forth, eliminating one threat then recovering before responding to the next threat.

\section{MICROBES AND IMMUNE REACTIONS}

There are a number of environmental conditions that may provoke and/or weaken the immune system-infections, cancer, allergens, stress, sleep deprivation, vaccinations, trauma, toxins, degenerative changes, molecular mimicry, low glutathione levels, increased oxidative stress, metal toxicity, elevated leptin levels and some medical treatments [1013]. Microbial effects are a major consideration for impacting neuronal functioning [14]. Thousands of peer-reviewed journal articles demonstrate the causal association between infections and mental illness and over 250 peer-reviewed scientific articles demonstrate the causal association between Lyme/tick-borne disease and mental illness [15].

Bacterial infections are recognized to be associated with many autoimmune diseases involving chronic inflammation and demyelination [16]. Possible modes of pathogenic action of bacteria include cytokines, toll-like receptor signaling, the interaction of heat shock proteins with the immune system, 
and nitric oxide. An auto-regulatory loop might exist in the interaction of bacteria with the host and in pathogenic signal processing [17]. Two common questions are whether trauma is directly from the infection or from the host's immune or other reactions to the infection and another and whether symptoms in the central nervous system (CNS) are a result of infection within the CNS or whether the infection is outside the CNS but immune effects are causing CNS symptoms? Parasite effects include cell penetration, toxin release and incorporation of parasite genes into the host genome. Host immune effects include cytokine release, antibodies, inflammation and other cellular responses. Sickness syndrome is a useful model to differentiate the symptoms commonly associated inflammation from the symptoms mediated by other processes. Sickness syndrome is mediated by the proinflammatory cytokines cascade with effects from interluken-1 (IL), IL-6, and tumor necrosis factor (TNF) [18]. Interferon treatment for Hepatitis $\mathrm{C}$ and other conditions is a good model for demonstrating inflammation mediated mental symptoms. Symptoms seen with interferon treatment include cognitive impairments, depression, anxiety, mania, irritability, impulsiveness, hostility, relapse of substance abuse and lassitude $[19,20]$.

\section{DISEASE PROGRESSION}

It is recognized chronic infections cause chronic stress, sleep disorders, cognitive impairments and chronic fatigue. Sleep disorders are commonly associated with chronic inflammatory diseases and chronic stress-related disorders. The best studied in this regard are rheumatoid arthritis, fibromyalgia, chronic fatigue syndromes and Lyme disease [21-23]. The bidirectional communication between the brain and the immune system contribute to inflammatory mediated disrupted sleep quality and conversely [24]. Cytokines produced by cells of the immune and nervous systems (particularly IL-1-beta and TNF-alpha) regulate sleep, signal neuroendocrine, autonomic, limbic and cortical areas of the CNS to affect neural activity and modify behaviors, hormone release and autonomic function [23]. To demonstrate the association between inflammation, chronic fatigue and sleep disturbances it has been demonstrated that sleep restriction increases IL-6 and pain-related symptoms in healthy volunteers and impaired sleep correlates with impaired immune functioning $[22,24]$. Growth hormone modulates adaptive immune response but growth hormone production is dependent upon the presence of delta sleep which is reduced in an inflammatory state [25]. Therefore, increasing delta sleep is therapeutic while disease progression is fostered by nonrestorative sleep and is associated with fatigue, cognitive impairments, pain and emotional symptoms [26]. The consequences of both non-restorative sleep and associated chronic stress reactions contribute to perpetuating the disease process and are associated with-decreased regenerative functioning, compromised immunity, oxidative stress and decreased resistance to infectious disease $[22,25,26]$.

\section{IMMUNE MEDIATED NEURODEGENERATIVE DISEASES}

Neurodegenerative diseases are a group of chronic, progressive disorders characterized by the gradual loss of neu- rons in discrete areas of the CNS. When neurodegenerative diseases are progressive uncontrolled inflammation drives disease progression [27]. Substantial evidence has documented a common inflammatory mechanism in various neurodegenerative diseases. It has been hypothesized that in the diseased CNS, interactions between damaged neurons and dysregulated, overactivated microglia create a vicious selfpropagating cycle causing uncontrolled, prolonged inflammation that drives the chronic progression of neurodegenerative diseases [27]. There is evidence with depression, Alzheimer's disease (AD), schizophrenia and epilepsy to support this position. A meta-analysis of cytokines in major depression including 24 studies reports significantly higher concentrations of the proinflammatory cytokines TNF-alpha and IL-6 in depressed subjects compared with control subjects [28]. A meta-analysis of cytokines in $\mathrm{AD}$ which reviewed 86 studies strengthens the clinical evidence that $A D$ is accompanied by an inflammatory response with particularly higher peripheral concentrations of IL-6, TNF, IL-1, transforming growth factor, IL-12 and IL-18 and higher CSF concentrations of transforming growth factor [29]. Hundreds of studies of schizophrenic illness in adults have documented immunological abnormalities in these patients. First-episode psychosis in children is associated with evidence of increased inflammation. Increasing evidence now suggests that the glia, cerebral vasculature, and the BBB may be involved which support the inflammatory theory of schizophrenia that was formulated over a 100 years ago [30]. There is a rapidly growing body of evidence that supports the involvement of inflammatory mediators in epilepsy-released by brain cells and peripheral immune cells - in both the origin of individual seizures and the epileptogenic process. Aspects of brain inflammation and immunity were first described and subsequently, it was demonstrated how seizures cause inflammation, and whether such inflammation, in turn, influences the occurrence and severity of seizures, and seizure-related neuronal death [31].

Immune mediated mechanisms include inflammatory and autoimmune mechanisms. The inflammatory mediated effects associated with neurodegenerative disease and include oxidative stress, excitotoxicity, proinflammatory cytokine effects and altered tryptophan metabolism.

\section{INFLAMMATORY MEDIATED MECHANISMS}

Oxidative stress and oxygen free radicals or activated oxygen has been implicated in diverse environmental stresses and appears to be a common contributor in neurodegenerative diseases [32].

Excitotoxicity and inadequate remethylation leads to increased homocysteine levels which are excitotoxic [33]. Elevated C-reactive protein levels are linked to a decline in executive function and frontal lobe damage. There is an association between elevated levels of high-sensitivity C-reactive protein, an indicator of low-grade inflammation, and decline in executive function [34].

Proinflammatory cytokines include Interferon alpha, IL1-beta and IL-6. Cytokine activation has been associated with psychiatric symptoms. For example, IL-6 is elevated in the cerebrospinal fluid of suicide attempters and is related to symptom severity, memory deficits and aggressiveness and 
IL-1-beta is associated with self-inflicted aggressive behavior and fatigue [35-37]. Besides cytokine effects, IL-1 receptor activation by systemic lipopolysaccharides has been demonstrated as one of the mechanisms by which environmentally driven immune system activation can trigger despair-like behavior in an animal model [38].

It has been proposed that parasites improve their survival by evolving mechanisms to change host behavior and some of these mechanisms are mediated by changes in serotonin and other monoamines [39]. Inflammation provoked by parasites impacts the conversion of tryptophan into serotonin. The kynurenine pathway is a major route of Ltryptophan catabolism into serotonin with a number of metabolites that include - kynurenic acid which is an $\mathrm{N}$-MethylD-aspartic acid (NMDA) antagonist (neuroprotective, unless excessive), quinolinic acid which is a NMDA agonist (neurotoxic). In an inflammatory state there is decreased serotonin \& a shift to quinolinic acid rather than kynurenic acid. The enzyme indoleamine 2,3-dioxygenase (IDO), which converts tryptophan into kynurenine and which is stimulated by proinflammatory cytokines, is implicated in the development of interferon--induced depressive symptoms, first by decreasing the serotonin availability to the brain and second by the induction of the kynurenine pathway resulting in the production of neurotoxic metabolites. In persistent infections associated with persistent inflammation, chronic activation of TNF-alpha stimulates interferon-gamma, which overactivates IDO, the rate-limiting enzyme for catabolism of tryptophan in the brain. Overactivated IDO causes neurotoxicity, and immune suppression of cytotoxic $T$ cells. Underactivation of IDO is known to cause autoimmune reactions, but it has recently been discovered that overactivated IDO causes autoimmune B cell antibody production [40]. CSF quinolinic acid is significantly elevated in a number of CNS infections including Borrelia burgdorferi (Bb), infection-dramatically in patients with CNS inflammation, less in encephalopathy. The presence of this known agonist of NMDA synaptic function; a receptor involved in learning, memory, and synaptic plasticity; may contribute to the neurologic and cognitive deficits seen in many Lyme disease patients [41].

\section{INFLAMMATION AND LYME/TICK-BORNE DIS- EASES}

Lyme disease, caused by the bacterium $B b$, has been recognized to cause multi-systemic signs and symptoms, including peripheral and central nervous system disease. Some immune mediated pathophysiology seen in Lyme/TickBorne Diseases (LYD/TBD) is a Failure to Shift from Th1 to Th2. Persisting immune activation causes the cytokine storm in chronic Lyme. In these patients, the innate immune system is not turned off by a series of specific immune peptides. Specific genetic types are more prone to this phenomenon [42].

Increased levels of the proinflammatory cytokines IL-6, IL-8, IL-12, IL-18 and interferon $\gamma$ and of the chemokines CXCL12 and CXCL13 have been reported in the CSF of patients with neurologic Lyme disease [43]. The magnitude of IL-6 in human serum and CSF has been shown to correlate with disease activity in neurologic Lyme disease [44]. Elevated levels of IL-6 can cause symptoms of fatigue and malaise, common to many infectious conditions as well as Lyme disease [45]. Borrelia species induce activation of IL17 production. The chemokine CXCL13 is a key regulator of $B$ cell recruitment to the cerebrospinal fluid in acute Lyme neuroborreliosis CSF CXCL13 and can be used as a diagnostic marker for infection [46-48].

$B b$ spirochetes express lipoproteins on the outer membrane of the Borrelia cell wall that is known to be proinflammatory. These lipoproteins attract neutrophils and have shown to be 50- to 500-fold more active inducers of cytokines and mitogens of B cells than lipoproteins of other organisms, such as Escherichia coli. Bacterial \& Borrelia lipoproteins can disseminate from the periphery to inflame the brain [43].

There are some other immune pathological processes associated with Lyme disease. The neuropsychiatric Herxheimer reaction appears to be an adverse immune reaction to treatment although the exact mechanism is not well clarified [49]. In the phase II AN1792 trial of active antiamyloid beta immunization against $\mathrm{AD}$, there were two patients fulfilling clinical $\mathrm{AD}$ criteria who were diagnosed with Lyme neuroborreliosis during screening who developed meningoencephalitis associated with destructive neuroinflammation apparently provoked by an interaction of the vaccine and the presence of Lyme neuroborreliosis [50]. The immune reactions seen in LYD/TBD are different from the immune reactions seen in chronic fatigue syndrome and this may partially be explained by the distinguishing cerebrospinal fluid protein complements that are seen in these patients when compared to healthy controls [51].

\section{MOLECULAR MIMICRY/AUTOIMMUNE MEDI- ATED MECHANISMS}

Paraneoplastic limbic encephalopathies and pediatric autoimmune diseases associated with strep (PANDAS) are good models to understand the effects of autoantibodies directed against intracellular neuronal antigens and the associated psychiatric symptoms. In paraneoplastic and nonparaneoplastic limbic encephalitis, voltage-gated potassium channel limbic encephalitis, Hashimoto's encephalopathy, anti-NMDA and other glutamate receptor encephalitis, encephalitis associated with gamma-aminobutyric acid signaling and systemic lupus erythematosus neurons are excited to death by autoantibodies resulting in neurotoxicity [52,53]. PANDAS is an interaction of a Streptococcal infection in a genetically susceptible individual at a young age which can result in obsessive compulsive disorder, tics and sometimes attention span difficulties. PANDAS is often comorbid with LYD/TBD and the broader categorization has been referred to as pediatric infection-triggered autoimmune neuropsychiatric disorders. Symptom flares follow a strep infection and correlate with increased antibody production [54-56].

Lyme surface antigens can cause molecular mimicry and associated autoimmune symptoms. $B b$ spirochetes surface glycolipids may elicit cross-reactive antibodies and $\operatorname{IgM~} B b$ flagella antibodies cross-reacted with neuronal antigens [43]. Anti-neural antibody reactivity has been demonstrated in patients with a history of Lyme borreliosis and persistent symptoms. Anti-neural antibody reactivity was found to be significantly higher in the Lyme patients with prior treatment 
and persistent symptoms (PLS) group than in the post-Lyme healthy and normal healthy groups [57]. Immunohistochemical analysis of PLS serum antibody activity demonstrated binding to cells in the central and peripheral nervous systems. The presence of anti-neural antibody reactivity in patients with PLS demonstrates 'objective immunologic abnormalities' and underscores the pathophysiologic nature of PLS and discredits the psychosomatic theory advanced by some as the cause of persisting symptoms [58]. Since immunologic abnormalities can be caused by an ongoing infectious process, a growing list of animal and human studies supports persistent infection in post-treatment Lyme patients. and current models of autoimmunity in other diseases suggest that persistent infection is required for the production of autoantibodies such as the anti-neural antibodies described by Chandra and colleagues; it is likely that persistent infection with the Lyme spirochete $B b$ may be driving production of these antibodies [59].

\section{CHRONIC INFECTIONS, LYME/TICK-BORNE DIS- EASE, IMMUNE EFFECTS AND AUTISM SPEC- TRUM DISORDER}

There has been recent attention to the association between chronic infections, LYD/TBD and autism spectrum disorders (ASD). Immune reactivity associated with these infections in the mother, fetus and child appear to adversely affect developing neural tissue and contribute to the pathophysiology associated with autism spectrum disorders. Possible pathophysiological mechanisms include both inflammatory processes as well as autoantibodies to developing neural tissue $[5,6,60,61]$.

During postnatal life, an intact BBB limits the entry of immune species into the brain. Lymphocytes, macrophages, various cytokines, and antibodies are generally maintained in the periphery. However, the blood-brain barrier is permeable during fetal development and can be compromised by infections and environmental exposures throughout life. The absence of a complete barrier allows immune components access to the brain. Individuals with autism show increased pro-inflammatory cytokines in the brain, as well as activation of microglia. Additionally, antibodies that target brain tissues have been described in both children with autism and their mothers. These immunological phenomena may interfere with normal brain development and function, potentially contributing to the development and/or symptoms of ASD [62].

One mouse model for cytokine mediated effects associated with ASD is demonstrated by maternal injection of IL-6 at different gestational stages is associated with different deficits associated with ASD [63]. Autoantibodies targeting brain proteins have been discovered in both children with autism and their mothers and circulating maternal autoantibodies directed toward fetal brain proteins are highly specific for autism. Additionally, data suggest there may be a defect in signaling pathways that are shared by the immune and central nervous systems. One model of autoimmune mediated effects associated with ASD is demonstrated by exposing rhesus monkeys to IgG from mothers of children with autism which results in the appearance of ASD symptoms [64]. In addition, antibodies that react to the 36, 37, 39, 61 and/or $73 \mathrm{kDa}$ bands on Western Blot testing are associated with provoking an immune reaction and contribute to causing autism. Reactivity to these bands is also associated with Borrelia burgdorferi and to a lesser degree to Bartonella henselae, Bartonella quintana, Mycoplasma, Chlamydia pneumonia and Streptococcus pneumoniae [5].

\section{CONCLUSION}

When looking at the clinical and basic science research on the subject articles it is apparent that persistent infection and associated inflammation and molecular mimicry mechanisms are associated with gradually increasing encephalopathy and gradually increasing mental symptoms. Cognitive symptoms begin as executive dysfunction and mild cognitive impairments and may gradually progress to dementia while emotional symptoms begin with insomnia, reduced frustration tolerance, irritability and dysthymia and may progress to anxiety disorders, depression, impulsivity and personality disorders and subsequently psychosis and/or suicidal and homicidal tendencies. Many of the neurological, cognitive and psychiatric symptoms associated with LYD/TBD appear to be mediated by immune mechanisms. Therefore greater interaction is needed between infectious disease specialists, immunologists and mental health practitioners.

\section{LIST OF ABBREVIATIONS}

$\begin{array}{ll}\mathrm{CNS} & =\text { Central nervous system } \\ \mathrm{IL} & =\text { Interleukin } \\ \mathrm{TNF} & =\text { Alzheimer's disease } \\ \mathrm{AD} & =\text { Blood brain barrier } \\ \mathrm{BBB} & =\text { N-Methyl-D-aspartic acid } \\ \mathrm{NMDA} & \text { Indoleamine 2,3-dioxygenase } \\ \mathrm{IDO} & =\text { Pediatric autoimmune diseases associated } \\ \mathrm{PANDAS} & \text { with strep } \\ \mathrm{PLS} & =\text { LYME patients with prior treatment and per- } \\ \mathrm{ASD} & =\text { Autism spectrum disorders }\end{array}$

\section{CONFLICT OF INTEREST}

The authors confirm that this article content has no conflicts of interest.

\section{ACKNOWLEDGEMENTS}

None declared.

\section{REFERENCES}

[1] Miklossy J. Alzheimer's disease - a neurospirochetosis. analysis of the evidence following Koch's and Hill's criteria. J Neuroinflammation 2011; 8: 90.

[2] Brown AS, Patterson PH. Maternal infection and schizophrenia: implications for prevention. Schizophr Bull 2011; 37: 284-90.

[3] Ling VJ, Lester D, Mortensen PB, Langenberg PW, Postolache TT. Toxoplasma gondii seropositivity and suicide rates in women. $J$ Nerv Ment Dis 2011; 199:440-4. 
[4] Mortensen CR, Becker DV, Ackerman JM, Neuberg SL, Kenrick DT. Infection breeds reticence: the effects of disease salience on self-perceptions of personality and behavioral avoidance tendencies. Psychol Sci 2010; 21: 440-7.

[5] Bransfield RC. Preventable cases of autism: relationship between chronic infectious diseases and neurological outcome Ped Health 2009; 3: 125-40.

[6] Bransfield RC, Wulfman JS, Harvey WT, Usman AI. The association between tick-borne infections, Lyme borreliosis and autism spectrum disorders Med Hypotheses 2008; 70: 967-74.

[7] Träskman-Bendz L, Westling S. The psychobiology of aggressive behaviour. Adv Health Econ Health Serv Res 2005; 16: 3-14.

[8] Knuesel I. Prenatal infection as driving force of aging-associated neurodegenerative diseases. Praxis (Bern 1994) 2011; 100: 299304.

[9] Daneman R, Rescigno M. The gut immune barrier and the bloodbrain barrier: are they so different? Immunity 2009; 31: 722-35.

[10] Kalinchuk AV, McCarley RW, Porkka-Heiskanen T, Basheer R. Sleep deprivation triggers inducible nitric oxide-dependent nitric oxide production in wake-active basal forebrain neurons. J Neurosci 2010; 30: 13254-64.

[11] Gardner RM, Nyland JF, Silbergeld EK. Differential immunotoxic effects of inorganic and organic mercury species in vitro. Toxicol Lett 2010; 198: 182-90.

[12] Jomova K, Valko M. Advances in metal-induced oxidative stress and human disease. Toxicology 2011; 283: 65-87.

[13] Aldabal L, Bahammam AS. Metabolic, endocrine, and immune consequences of sleep deprivation. Open Respir Med J 2011; 5: 3143.

[14] Kristensson K. Microbes' roadmap to neurons. Nat Rev Neurosci 2011; 12: 345-57.

[15] Bransfield R. Neuropsychiatric Lyme disease: pathophysiology, assessment and treatment. In: $2^{\text {nd }}$ International Lyme and associated diseases society European meeting, Augsburg, Germany; May, 2011

[16] Chervonsky AV. Influence of microbial environment on autoimmunity. Nat Immunol 2010; 11: 28-35.

[17] Chen K, Huang J, Gong W, Iribarren P, Dunlop NM, Wang JM. Toll-like receptors in inflammation, infection and cancer. Int Immunopharmacol 2007; 7: 1271-85.

[18] Musselman DL, Lawson DH, Gumnick JF, et al. Paroxetine for the prevention of depressioninduced by high-dose interferon alfa. $\mathrm{N}$ Engl J Med 2001; 344: 961-6.

[19] Constant A, Castera L, Dantzer R, et al. Mood alterations during interferon-alfa therapy in patients with chronic hepatitis $\mathrm{C}$ : evidence for an overlap between manic/hypomanic anddepressive symptoms. J Clin Psychiatry 2005; 66: 1050-7.

[20] Hong BA, North CS, Pollio DE, et al. The use of psychoeducation for a patient with hepatitis $\mathrm{C}$ and psychiatric illness in preparation for antiviral therapy: a case report and discussion. J Clin Psychol Med Settings 2011; 18: 99-107.

[21] Greenberg HE, Ney G, Scharf SM, Ravdin L, Hilton E. Sleep quality in Lyme disease. Sleep 1995; 18: 912-6.

[22] Moldofsky H. Sleep and the immune system. Int J Immunopharmacol 1995; 17: 649-54.

[23] Lorton D, Lubahn CL, Estus C, et al. Bidirectional communication between the brain and the immune system: implicationsfor physiological sleep and disorders with disrupted sleep. Neuroimmunomodulation 2006; 13: 357-74.

[24] Haack M, Sanchez E, Mullington JM. Elevated inflammatory markers in response to prolonged sleep restriction are associated with increased pain experience in healthy volunteers. Sleep 2007; 30: $1145-52$.

[25] Kelley KW. The role of growth hormone in modulation of the immune response. Ann N Y Acad Sci. 1990; 594: 95-103.

[26] Schmidt-Wilcke T, Clauw DJ. Fibromyalgia: from pathophysiology to therapy. Nat Rev Rheumatol 2011; 7(9): 518-27.

[27] Gao HM, Hong JS. Why neurodegenerative diseases are progressive: uncontrolled inflammation drives disease progression. Trends Immunol 2008; 29: 357-65.

[28] Dowlati Y, Herrmann N, Swardfager W, et al. A meta-analysis of cytokines in major depression. Biol Psychiatry 2010; 67: 446-57.

[29] Swardfager W, Lanctôt K, Rothenburg L, Wong A, Cappell J, Herrmann N. A meta-analysis of cytokines in Alzheimer's disease. Biol Psychiatry 2010; 68: 930-41.
[30] Tatiana Falcone T, Carlton E, Franco K, Janigro D. Inflammation, psychosis, and the brain. Psychiatr Times 2009; 26.

[31] Vezzani A, French J, Bartfai T, Baram TZ. The role of inflammation in epilepsy. Nat Rev Neurol 2011;7: 31-40.

[32] Naik E, Dixit VM. Mitochondrial reactive oxygen species drive proinflammatory cytokine production. J Exp Med 2011; 208: 41720.

[33] Bleich S, Degner D, Sperling W, Bönsch D, Thürauf N, Kornhuber J. Homocysteine as a neurotoxin in chronic alcoholism. Prog Neuropsychopharmacol Biol Psychiatry 2004; 28: 453-64.

[34] Wersching H, Duning T, Lohmann H, et al. Serum C-reactive protein is linked to cerebral microstructural integrity and cognitive function .Neurology 2010; 74: 1022-9.

[35] Lindqvist D, Janelidze S, Hagell P, et al. Interleukin-6 is elevated in the cerebrospinal fluid of suicide attempters and related to symptom severity. Biol Psychiatry 2009; 66: 287-92.

[36] Westling S, Ahrén B, Träskman-Bendz L, Brundin L. Increased IL$1 \beta$ reactivity upon a glucose challenge in patients with deliberate self-harm. Acta Psychiatr Scand 2011; 124(4): 301-6.

[37] Corwin EJ, Bozoky I, Pugh LC, Johnston N. Interleukin-1beta elevation during the postpartum period. Ann Behav Med. 2003; 25: 41-7.

[38] Zhu CB, Lindler KM, Owens AW, Daws LC, Blakely RD, Hewlett WA. Interleukin-1 receptor activation by systemic lipopolysaccharide induces behavioral despair linked to MAPK regulation of CNS serotonin transporters. Neuropsychopharmacology 2010; 35: 251020 .

[39] Sergiev VP. Change of host's behavior including man under the influence of parasites. Zh Mikrobiol Epidemiol Immunobiol 2010; 3: 108-14.

[40] Wichers MC, Koek GH, Robaeys G, Verkerk R, Scharpé S, Maes $\mathrm{M}$. IDO and interferon-alpha-induced depressive symptoms: a shift in hypothesis from tryptophan depletion to neurotoxicity. Mol Psychiatry $2005 ; 10: 538-44$.

[41] Halperin JJ, Heyes MP. Neuroactive kynurenines in Lyme borreliosis. Neurology 1992; 42: 43-50.

[42] Newell MK, Tobin RP, Cabrera JH, et al. TLR-mediated B cell activation results in ectopic CLIP expression that promotes B celldependent inflammation. J Leukoc Biol 2010; 88: 779-89.

[43] Fallon BA, Levin ES, Schweitzer PJ, Hardesty D. Inflammation and central nervous system Lyme disease. Neurobiol Dis 2010; 37: 534-41.

[44] Weller M, Stevens A, Sommer N, Wiethölter H, Dichgans J. Cerebrospinal fluid interleukins, immunoglobulins, and fibronectin in neuroborreliosis. Arch Neurol 1991; 48: 837-41.

[45] Pachner AR, Amemiya K, Delaney E, O'Neill T, Hughes CA, Zhang WF. Interleukin-6 is expressed at high levels in the CNS in Lyme neuroborreliosis. Neurology 1997; 49: 147-52.

[46] Rupprecht TA, Plate A, Adam M, et al. The chemokine CXCL13 is a key regulator of $\mathrm{B}$ cell recruitment to the cerebrospinal fluid in acute Lyme neuroborreliosis. J Neuroinflammation 2009; 6: 42.

[47] Wutte N, Berghold A, Loffler S, et al. CXCL13 chemokine in pediatric and adult neuroborreliosis. Acta Neurol Scand 2011 124(5): 321-8.

[48] Tjernberg I, Henningsson AJ, Eliasson I, Forsberg P, Ernerudh J. Diagnostic performance of cerebrospinal fluid chemokine CXCL13 and antibodies to the C6-peptide in Lyme neuroborreliosis. J Infect 2011; 62: 149-58.

[49] Maloy AL, Black RD, Segurola RJ Jr. Lyme disease complicated by the Jarisch-Herxheimer reaction. J Emerg Med 1998; 16: 437-8.

[50] Andreasen N, Blennow K, Zetterberg H. Neuroinflammation screening in immunotherapy trials against Alzheimer's disease. Int J Alzheimers Dis 2010; 2010: 638379.

[51] Schutzer SE, Angel TE, Liu T, et al. Distinct cerebrospinal fluid proteomes differentiate post-treatment lyme disease from chronic fatigue syndrome. PLoS One 2011; 6: e17287.

[52] Kayser MS, Dalmau J. Psychiatric Presentations of Autoimmune Encephalopathies. Psychiatr Times 2010; 27.

[53] Moscato EH, Jain A, Peng X, Hughes EG, Dalmau J, BaliceGordon RJ. Mechanisms underlying autoimmune synaptic encephalitis leading to disorders of memory, behavior and cognition: insights from molecular, cellular and synaptic studies. Eur J Neurosci 2010; 32: 298-309.

[54] Schneider RK, Robinson MJ, Levenson JL. Psychiatric presentations of non-HIV infectious diseases. Neurocysticercosis, Lyme disease, and pediatric autoimmune neuropsychiatric disorder asso- 
ciated with streptococcal infection. Psychiatr Clin North Am 2002; 25: 1-16.

[55] Ercan TE, Ercan G, Severge B, Arpaozu M, Karasu G. Mycoplasma pneumoniae infection and obsessive-compulsive disease: a case report. J Child Neurol 2008; 23: 338-40.

[56] Leah E. Neuropsychiatric disorders: Neurons excited to death by SLE autoantibodies. Nat Rev Rheumatol 2011; 7: 1.

[57] Chandra A, Wormser GP, Klempner MS, et al. Anti-neural antibody reactivity in patients with a history of Lyme borreliosis and persistent symptoms. Brain Behav Immun 2010; 24: 1018-24.

[58] Hassett AL, Radvanski DC, Buyske S, Savage SV, Sigal LH. Psychiatric comorbidity and other psychological factors in patients with chronic Lyme disease. Am J Med 2009; 122: 843-50.

[59] Stricker RB, Johnson L. Letter to the editor re "Anti-neural antibody reactivity in patients with a history of Lyme borreliosis and persistent symptoms" by Chandra et al. Brain Behav Immun. 2010; 24:1025; author reply 1026 .
[60] Nicolson GL, Gan R, Nicolson NL, Haier J. Evidence for Mycoplasma, Chlamydia pneunomiae and HHV-6 co-infections in the blood of patients with autism spectrum disorders. J Neurosci Res 2007; 85: 1143-8.

[61] Nicholson G. Chronic bacterial and viral infections in neurodegenerative and neurobehavioral diseases. Lab Med 2008; 39: 291-9.

[62] Goines P, Van de Water J. The immune system's role in the biology of autism. Curr Opin Neurol 2010; 23: 111-7.

[63] Smith SE, Li J, Garbett K, Mirnics K, Patterson PH. Maternal immune activation alters fetal brain development through interleukin-6. J Neurosci 2007; 27: 10695-702.

[64] Martin LA, Ashwood P, Braunschweig D, Cabanlit M, Van de Water J, Amaral DG. Stereotypies and hyperactivity in rhesus monkeys exposed to IgG from mothers of children with autism. Brain Behav Immun 2008; 22: 806-16.

(C) Robert C. Bransfield; Licensee Bentham Open.

This is an open access article licensed under the terms of the Creative Commons Attribution Non-Commercial License (http://creativecommons.org/licenses/by-nc/3.0/) which permits unrestricted, non-commercial use, distribution and reproduction in any medium, provided the work is properly cited. 